\title{
Testes de equilíbrio e mobilidade funcional na predição e prevenção de riscos de quedas em idosos
}

\section{Balance and mobility functional tests in the prediction and prevention of risks of falls in elderly}

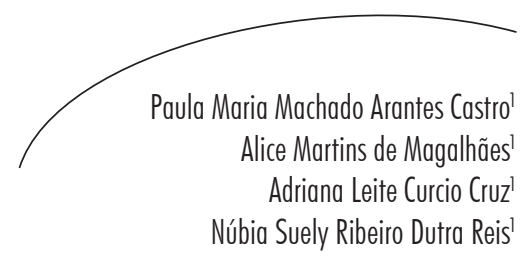

Resumo

Introdução: A prevenção da ocorrência de quedas em idosos é um desafio para os profissionais da área da saúde. Um dos aspectos mais relevantes para tal prevenção é a detecção precoce de fatores de risco para quedas. Dentre estes, destaca-se o declínio do equilíbrio e da mobilidade funcional. Objetivos: Avaliar a ocorrência de quedas e seus fatores associados e identificar os pontos de corte de testes de equilíbrio e mobilidade funcional mais adequados para identificar idosos ativos na comunidade com risco de quedas. Metodologia: Estudo transversal composto por 127 idosos ativos na comunidade de Itabira-MG, avaliados quanto às características clínicas e sociodemográficas e ao equilíbrio e mobilidade funcional (testes sentado para de pé, semi-tandem, step teste e velocidade da marcha de seis metros). Resultados: A prevalência de quedas no ano anterior foi $25,2 \%$, sendo $8,6 \%$ para quedas recorrentes. A ocorrência de quedas apresentou associação apenas com a variável "gênero", sendo maior a chance de cair entre as mulheres. Os pontos de corte para os testes sentado para de pé, step teste e velocidade da marcha foram 8,5 segundos, 7,5 segundos e $0,98 \mathrm{~m} / \mathrm{s}$, respectivamente. Conclusão: Os resultados demonstraram ser importante adequar os pontos de corte dos testes de equilíbrio e mobilidade ao perfil da população avaliada. Sem essa adequação, os testes têm sua sensibilidade e especificidade comprometidas na identificação de idosos com risco de cair.

\section{Abstract}

Introduction: The prevention of falls in the elderly is a challenge for health professionals. One of the most important aspects for such prevention is early detection of risk factors for falls. Among these, we highlight the declining balance and functional mobility. Objectives: To evaluate the occurrence of falls and associated factors and identify the most suitable cutoff points of balance and functional mobility tests to identify active seniors in the community at risk of falling. Methods: Cross-sectional study comprising 127 active seniors in the community in the city of Itabira-MG, Brazil, evaluated for clinical and demographic characteristics and balance and

Palavras-chave: Idoso. Equilíbrio Postural. Mobilidade Funcional. Acidentes por Quedas.

Key words: Elderly. Postural Balance. Functional Mobility. Accidental Falls.

\footnotetext{
Fundação Comunitária e Ensino Superior de Itabira - Funcesi. Itabira-MG, Brasil.
} 
functional mobility tests (seated to standing, semi-tandem, step test and gait speed of six meters). Results: Prevalence of falls in the previous year was $25.2 \%$, and $8.6 \%$ for recurrent falls. Occurrence of falls was associated only with the gender variable, with a higher chance of falling among women. The cutoff points for tests seated to standing, step test and gait speed was 8.5 seconds, 7.5 seconds and $0.98 \mathrm{~m} / \mathrm{s}$, respectively. Conclusion: The results proved to be important to match the cutoff tests of balance and mobility profile of the population. Without this adjustment, tests have sensitivity and specificity in identifying compromised elderly at risk of falling.

\section{INTRODUÇÃO}

As quedas constituem o principal problema de cuidado à saúde da população idosa. Aproximadamente $30 \%$ dos indivíduos com mais de 65 anos de idade caem ao menos uma vez por ano, dos quais a metade de forma recorrente. ${ }^{1} \mathrm{~A}$ cada ano, o Sistema Único de Saúde (SUS) tem gastos crescentes com o tratamento de problemas decorrentes das quedas. ${ }^{2}$ Seu custo social éimenso e torna-se maior quando o idoso tem diminuição da autonomia e da independência ou passa a necessitar de institucionalização. ${ }^{3}$ Dentre as principais consequências das quedas encontramse as fraturas, o aumento da dependência, o medo de novas quedas, a restrição de atividades, a institucionalização, o aumento da morbidade e altos índices de mortalidade. ${ }^{4}$ As quedas nos idosos podem também repercutir entre os cuidadores e familiares, que devem se mobilizar em torno de cuidados especiais, adaptando sua rotina em função da recuperação ou adaptação do idoso após a queda. ${ }^{1,2,5}$

Vários fatores de risco para a ocorrência de quedas em idosos têm sido relatados, como diminuição da força muscular, alterações visuais, redução do equilíbrio, alterações da marcha, artrites, déficit cognitivo e uso de quatro ou mais medicamentos, além de outros fatores biológicos, ambientais, comportamentais e sociais. ${ }^{6,7}$ As quedas resultam da interação desses fatores e não apenas do efeito aditivo de comorbidades e incapacidades físicas. ${ }^{7}$ A identificação precoce desses fatores de risco é extremamente importante para a prevenção da ocorrência de quedas. ${ }^{8,9}$ Dentre os fatores que aumentam o risco de quedas nos idosos, a deterioração do equilíbrio e da mobilidade funcional são considerados muito importantes. ${ }^{10-12}$

Vários testes têm sido descritos e utilizados para avaliar o equilíbrio e mobilidade funcional dos idosos, com o objetivo de determinar o risco de quedas. ${ }^{13,14}$ Testes como sentado para de pé, equilíbrio estático na posição semi-tandem, step teste e teste da velocidade da marcha são bastante utilizados na prática clínica, considerando a facilidade de aplicação, necessidade de poucos recursos e boa sensibilidade e especificidade. ${ }^{13,14}$

Entretanto, os pontos de cortes para estes testes mais apropriados para idosos brasileiros, vivendo na comunidade, ainda não foram estabelecidos. Esse conhecimento contribuirá para a identificação precoce do risco de cair e assim, a prevenção da ocorrência de quedas. Portanto, os objetivos deste estudo foram avaliar a ocorrência de quedas e seus fatores associados e identificar os pontos de corte de testes de equilíbrio e mobilidade funcional mais adequados para identificar idosos ativos na comunidade com risco de quedas.

\section{METODOLOGIA}

\section{Delineamento e participantes}

Estudo de delineamento transversal, com amostra composta por 127 idosos residentes 
na comunidade da cidade de Itabira-MG. Foi realizado o cálculo de tamanho amostral, no qual se consideraram nível de significância estatística de $\alpha=0,05$, intervalo de confiança de $95 \%$ e prevalência de quedas na população idosa descrita no Estudo Saúde, Bem-estar e Envelhecimento (SABE). ${ }^{15}$ Os idosos foram recrutados do Projeto Vida Ativa da prefeitura de Itabira-MG, Associação da Aposvale e Grupo da Terceira Idade da Unimed-Itabira-MG. Esses grupos dos quais os idosos foram recrutados são de convivência para atividades sociorecreativas e exercícios físicos, com supervisão de educadores físicos e fisioterapeuta.

Para serem incluídos no presente estudo, os idosos deveriam atender aos seguintes critérios: idade igual ou superior a 65 anos, serem capazes de deambular seis metros de forma independente, viverem na comunidade e assinar o Termo de Consentimento Livre Esclarecido. Os critérios de exclusão foram: presença de déficit de compreensão que limitasse a execução dos movimentos por meio de comandos verbais; presença de doença neurológica (ex: doença de Parkinson e acidente vascular encefálico); ter realizado no último ano qualquer intervenção cirúrgica, iniciar algum outro tipo de atividade física diferente ou apresentar algum agravo à saúde que comprometesse o aparelho locomotor durante o período de acompanhamento no estudo.

\section{Instrumentos}

Foram utilizados os testes velocidade de marcha de seis metros, sentado para de pé, equilíbrio estático na posição semi-tandem e o step teste. Adicionalmente, utilizou-se o Questionário Internacional de Atividade Física (IPAQ) para avaliação do nível de atividade.

O IPAQ é um questionário que permite estimar o tempo semanal gasto em atividades físicas, em diferentes situações do cotidiano. No Brasil, os estudos têm demonstrado que este é um instrumento com boa estabilidade de medidas, precisão aceitável e validade para uso em estudos com idosos brasileiros. ${ }^{16-18}$

O teste sentado para de pé consiste em levantar e sentar de uma cadeira determinado número de vezes. Esse varia entre os estudos, mas normalmente é entre uma e cinco vezes. Tiedemann ${ }^{13}$ evidenciou que o teste realizado com cinco repetições tem maior poder de discriminar idosos com e sem história de quedas, em relação aos testes realizados com número menor de repetições.

O step teste avalia requerimentos para a marcha e para subir e descer escadas e, uma vez que envolve transferência de peso de um pé para o outro, é uma medida de estabilidade médio-lateral. ${ }^{19} \mathrm{O}$ teste consiste em colocar alternadamente os pés sobre um degrau de $18 \mathrm{~cm}$ de altura e $40 \mathrm{~cm}$ de profundidade..$^{13} \mathrm{O}$ tempo gasto para completar a tarefa compreende o resultado do teste.

O teste de equilíbrio estático na posição semitandem é uma medida de estabilidade lateral e tem sido utilizado para identificar idosos com risco de quedas. ${ }^{13} \mathrm{O}$ indivíduo é solicitado a ficar na posição semi-tandem e o tempo de permanência sem perda de equilíbrio é registrado.

\section{Procedimentos de coleta}

Antes da realização da pesquisa, foi realizado estudo piloto para análise da confiabilidade intraexaminador das medidas dos testes de equilíbrio e mobilidade funcional. Observouse o período de uma semana entre os dois testes. Os valores de coeficiente de correlação intraclasse foram superiores a 0,90 , indicando boa confiabilidade. ${ }^{20}$ Os idosos foram contatados em visita aos grupos Vida Ativa, Aposvale e Unimed de Itabira-MG quando foi feita uma triagem inicial. Após receberem informações detalhadas a respeito do estudo, os idosos que atenderam aos critérios de inclusão e exclusão e 
que tiveram interesse em participar assinaram o Termo de Consentimento Livre e Esclarecido. A coleta de dados foi realizada no período de abril a junho de 2013. A pesquisa foi aprovada pelo Comitê de Ética em Pesquisa da Fundação Comunitária de Ensino Superior de Itabira, sob parecer $n^{\circ}$ 002/2013.

Aqueles que se disponibilizaram a participar da pesquisa foram convidados para avaliação, que ocorreu no local do grupo Vida Ativa e Aposvale. Os idosos do grupo de terceira idade da UnimedItabira-MG foram convidados a participar da avaliação na Fundação Comunitária de Ensino Superior de Itabira.

Realizou-se inicialmente a coleta de dados sociodemográficos e clínicos, como dados vitais sendo pressão arterial e frequência cardíaca, número de comorbidades, utilização de medicamentos, ocorrência de quedas e número de hospitalizações.

As avaliações foram realizadas sempre por duas examinadoras previamente treinadas; sendo que a examinadora 1 realizava a anamnese e a examinadora 2 aplicava os testes e o questionário IPAQ. Os testes sentado para de pé, equilíbrio estático na posição semi-tandem e o step teste foram realizados de acordo com os procedimentos descritos por Tiedemann. ${ }^{13}$ Para o teste sentado para de pé, foi utilizada uma cadeira fixa sem braços e com $45 \mathrm{~cm}$ de altura. Os participantes foram instruídos a levantarem e sentarem da cadeira cinco vezes, o mais rápido possível. Durante a realização do teste, os participantes foram orientados a manter os braços cruzados na frente de corpo.

Para a realização do teste de equilíbrio estático na posição semi-tandem, foi solicitado ao participante que permanecesse em pé, com os pés separados lateralmente por $2,5 \mathrm{~cm} \mathrm{e}$ com o calcanhar do pé que estivesse na frente afastado $2,5 \mathrm{~cm}$ do hálux do pé que estivesse atrás. $\mathrm{O}$ participante poderia escolher qual pé ficaria na frente e foi orientado a tentar permanecer 30 segundos nesta posição, com os olhos fechados. Foi registrado o tempo que ele foi capaz de manter-se na posição sem dar um passo ou abrir os olhos.

Em seguida, o participante foi solicitado a realizar o step teste, sendo o mesmo instruído a colocar alternadamente o pé direito e esquerdo sobre um degrau, o mais rápido possível. O pé deveria ser colocado completamente sobre o degrau. Foi registrado o tempo que o participante gastou para colocar os pés, alternando entre o direito e esquerdo, oito vezes.

Para avaliação da velocidade da marcha, o participante foi solicitado a deambular uma distância de seis metros. ${ }^{21,22}$ Foram descartados os períodos de aceleração e desaceleração (1,5 metros iniciais e finais). A velocidade da marcha era autosselecionada e a considerada confortável e usual para o participante.

\section{Análise estatística}

A análise estatística foi conduzida no programa Statistical Package for the Social Sciences (SPSS) versão 17.0 para Windows (todas as variáveis foram analisadas nesse pacote estatístico). Foi realizada análise descritiva dos dados, com cálculo de frequência para variáveis categóricas e de média e desvio-padrão para variáveis intervalares. Para avaliação da associação entre as variáveis categóricas e a ocorrência de quedas, foi utilizado o teste Qui-quadrado. Foram construídas curvas ROC para cada uma das variáveis dependentes, para avaliação da sensibilidade e especificidade destas e, assim, determinação do ponto de corte. O nível de significância foi estabelecido em $\mathrm{p}=0,05$. 


\section{RESULTADOS}

A amostra foi constituída por 127 idosos residentes na comunidade $(73,09 \pm 6,87$ anos, mínimo 65 e máximo 95 anos), sendo 62 homens e 65 mulheres. Nessa amostra, 32 idosos $(25,2 \%)$ relataram ter caído pelo menos uma vez no ano anterior. As características clínicas e sociodemográficas da amostra estão descritas na tabela 1.

Tabela 1. Características clínicas da amostra. Itabira-MG, 2013.

\begin{tabular}{|c|c|c|}
\hline Categoria & Classificação & $\%$ \\
\hline \multirow{4}{*}{ Comorbidades } & Nenhuma & 6,30 \\
\hline & Uma a duas & 66,90 \\
\hline & Três a quatro & 22,00 \\
\hline & Cinco a seis & 4,70 \\
\hline \multirow{3}{*}{ Medicamentos } & Nenhum & 5,50 \\
\hline & Um a três & 51,20 \\
\hline & Quatro ou mais & 43,41 \\
\hline \multirow{2}{*}{ Dor } & Sem dor & 24,20 \\
\hline & Com dor & 75,80 \\
\hline \multirow{3}{*}{ IPAQ } & NAF- baixo & 25,80 \\
\hline & NAF- moderado & 58,60 \\
\hline & NAF- vigoroso & 14,80 \\
\hline \multirow{5}{*}{ Prática de AF } & Irregular & 7,80 \\
\hline & Regular 2x/ semana & 32,80 \\
\hline & Regular 3x/ semana & 14,00 \\
\hline & Regular $4 \mathrm{x} /$ semana & 18,80 \\
\hline & Regular 5x/ semana & 25,60 \\
\hline \multirow{3}{*}{ Quedas } & Nenhuma & 74,80 \\
\hline & Uma & 16,50 \\
\hline & Duas ou mais & 8,70 \\
\hline \multirow{4}{*}{ Medo de cair } & Não & 39,80 \\
\hline & Pouco & 21,10 \\
\hline & Moderado & 20,30 \\
\hline & Muito & 18,00 \\
\hline \multirow{2}{*}{ Restrição na atividade } & Não & 70,30 \\
\hline & $\operatorname{Sim}$ & 29,70 \\
\hline
\end{tabular}

$\mathrm{IPAQ}=$ questionário internacional de atividade física; $\mathrm{NAF}=$ nível de atividade física; $\mathrm{AF}=$ atividade física. 
Em relação às causas das quedas, a mais frequentemente relatada pelos idosos foi o tropeço $(38,9 \%)$, seguida de desequilíbrio $(19,4 \%)$, escorregão $(13,9 \%)$ e tonteira $(13,9 \%)$. Apenas 5,5\% dos idosos relataram que caíram devido ao falseio dos membros inferiores. Adicionalmente, 5,5\% relataram ter caído devido à ingestão exagerada de álcool e 5,5\% caíram em situação de risco (subindo em árvore e correndo atrás de animais). Entre os idosos que caíram, 11\% tiveram sua queda relacionada a um comportamento de risco. Destes, nenhum apresentava alteração nos testes de equilíbrio e mobilidade funcional. Já em relação aos demais idosos que caíram, por causa não relacionada a comportamento de risco, 64,3\% apresentavam alteração em pelo menos um dos testes.

O desempenho dos idosos nos testes de equilíbrio e mobilidade funcional está apresentado na tabela 2 .

Tabela 2. Desempenho do teste de equilíbrio e mobilidade (média \pm desvio-padrão). Itabira-MG, 2013.

\begin{tabular}{cccc}
\hline Teste & Idoso com HQ & Idoso sem HQ & Total \\
\hline Semi-tandem (s) & $28,69 \pm 5,16$ & $29,81 \pm 1,37$ & $29,53 \pm 2,86$ \\
Sentado para de pé (s) & $9,38 \pm 2,43$ & $8,87 \pm 2,27$ & $9,0 \pm 2,3$ \\
Step $(\mathrm{s})$ & $8,13 \pm 2,32$ & $7,97 \pm 2,18$ & $8,01 \pm 2,2$ \\
Velocidade $(\mathrm{m} / \mathrm{s})$ & $1,02 \pm 0,38$ & $1,09 \pm 0,24$ & $1,08 \pm 0,23$ \\
\hline
\end{tabular}

$\mathrm{HQ}=$ história de quedas.

De acordo com os resultados, não houve associação entre o nível de atividade física e ocorrência de quedas nessa amostra. Também não foi verificada associação entre esta variável e o medo de cair, restrição de atividades e autopercepção de saúde (tabela 3). Adicionalmente, foi verificada maior ocorrência de quedas entre as mulheres, em comparação aos homens $(\mathrm{p}=0,045)$. 
Tabela 3. Associação entre as características clínicas e sociodemográficas e história de quedas. ItabiraMG, 2013.

\begin{tabular}{|c|c|c|c|c|c|}
\hline Variável & $\begin{array}{c}\text { História de } \\
\text { Quedas }\end{array}$ & & Categorias & & Valor $\mathrm{p}$ \\
\hline \multirow{3}{*}{ Sexo } & & Masculino & Feminino & & \multirow{3}{*}{$0,045 \%$} \\
\hline & Não & $53,7 \%$ & $34,4 \%$ & & \\
\hline & Sim & $46,3 \%$ & $65,6 \%$ & & \\
\hline \multirow{3}{*}{ Medo de cair } & & $\begin{array}{c}\text { Moderado / } \\
\text { muito }\end{array}$ & Pouco & Não & \multirow{3}{*}{$0,887 \%$} \\
\hline & Não & $36,8 \%$ & $22,1 \%$ & $41,1 \%$ & \\
\hline & Sim & $43,8 \%$ & $18,8 \%$ & $37,5 \%$ & \\
\hline \multirow{4}{*}{ IPAQ } & & NAF & NAF Moderado & NAF Viororoso & \multirow{4}{*}{$0,828 \%$} \\
\hline & & Baixo & & & \\
\hline & Não & $27,4 \%$ & $57,9 \%$ & $14,7 \%$ & \\
\hline & Sim & $21,9 \%$ & $62,5 \%$ & $15,6 \%$ & \\
\hline \multirow{3}{*}{$\begin{array}{c}\text { Sentado para de } \\
\text { pé* }\end{array}$} & & Sem risco & Com risco & & \multirow{3}{*}{$0,391 \%$} \\
\hline & Não & $87,4 \%$ & $12,6 \%$ & & \\
\hline & Sim & $81,3 \%$ & $18,8 \%$ & & \\
\hline \multirow{3}{*}{ Step teste* } & & Sem risco & Com risco & & \multirow{3}{*}{$0,434 \%$} \\
\hline & Não & $78,9 \%$ & $21,1 \%$ & & \\
\hline & Sim & $87,5 \%$ & $12,5 \%$ & & \\
\hline \multirow{3}{*}{ Velocidade* } & & Sem risco & Com risco & & \multirow{3}{*}{$0,304 \%$} \\
\hline & Não & $61,1 \%$ & $38,9 \%$ & & \\
\hline & Sim & $50 \%$ & $50 \%$ & & \\
\hline \multirow{3}{*}{$\begin{array}{c}\text { Autopercepção } \\
\text { da saúde }\end{array}$} & & Boa & Excelente & Moderada & \multirow{3}{*}{$0,691 \%$} \\
\hline & Não & $52,6 \%$ & $12,6 \%$ & $31,6 \%$ & \\
\hline & Sim & $46,9 \%$ & $18,8 \%$ & $28,1 \%$ & \\
\hline \multirow{3}{*}{$\begin{array}{l}\text { Restrição na } \\
\text { participação }\end{array}$} & & Sim & Não & & \multirow{3}{*}{$0,637 \%$} \\
\hline & Não & $71,6 \%$ & $28,4 \%$ & & \\
\hline & Sim & $65,6 \%$ & $34,4 \%$ & & \\
\hline
\end{tabular}

$\mathrm{NAF}=$ nível de atividade física; $*$ presença do fator de risco para o teste de acordo com o ponto de corte de Tiedemann et al. ${ }^{13}$ 
Em relação à analise da curva ROC dos testes de equilíbrio e mobilidade, verificou-se que os pontos de corte mais apropriados para a identificação dos idosos com história de quedas estão apresentados na tabela 4. Para a amostra do estudo, o teste semi-tandem não apresentou valores de sensibilidade e especificidade adequados, uma vez que os indivíduos apresentaram desempenho muito superior neste teste.

Tabela 4. Ponto de corte dos testes de equilíbrio e mobilidade. Itabira-MG, 2013.

\begin{tabular}{lcc|cc|c}
\hline Variável & $\mathrm{n}$ & $\begin{array}{c}\text { Ponto de corte } \\
\text { (segundos) }\end{array}$ & $\begin{array}{c}\text { Sensibilidade } \\
(\%)\end{array}$ & $\begin{array}{c}\text { Especificidade } \\
(\%)\end{array}$ & $\begin{array}{c}\text { Área sob a } \\
\text { curva }\end{array}$ \\
\hline $\begin{array}{l}\text { Sentado para de } \\
\text { pé (s) }\end{array}$ & 127 & 8,50 & 59,4 & 48,4 & 0,563 \\
$\begin{array}{l}\text { Step teste (s) } \\
\begin{array}{l}\text { Velocidade } \\
\text { (m/s) }\end{array}\end{array}$ & 127 & 7,50 & 65,4 & 51,6 & 0,528 \\
\hline
\end{tabular}

\section{DISCUSSÃO}

A ocorrência de quedas no presente trabalho $(25,2 \%)$ foi inferior à descrita em alguns estudos, tanto em relação à ocorrência de uma queda, como em relação a quedas recorrentes. ${ }^{1,23}$ Prevalências de $32,1 \%$ e $34,8 \%$ foram relatadas previamente. ${ }^{1,23}$ Em relação às quedas recorrentes, enquanto no presente estudo $8,7 \%$ dos idosos caíram mais de uma vez no ano anterior, Siqueira et al. ${ }^{1}$ relataram prevalência de $15,7 \%$. Por outro lado, pesquisas recentes com idosos brasileiros relataram prevalências de quedas semelhantes à do presente estudo. Siqueira et al. ${ }^{24}$ avaliaram 1.616 idosos e encontraram prevalência de 27,6\%. Adicionalmente, Coimbra et al. ${ }^{25}$ encontraram prevalência de $27,1 \%$ para uma queda no ano anterior e 8,7\% para ocorrência de quedas recorrentes no mesmo período.

Verificou-se, neste estudo, que grande parte de sua amostra apresentava pelo menos uma comorbidade. Adicionalmente, observou-se que a utilização de medicamentos regularmente também foi muito frequente. Esses dados estão de acordo com estudos prévios sobre envelhecimento. ${ }^{26,27}$ A presença de algumas doenças e o uso de múltiplos medicamentos são considerados fatores de risco para ocorrência de quedas em idosos. ${ }^{28,29}$ Neste estudo, 43,4\% dos idosos usavam quatro ou mais medicamentos regularmente, indicando que uma parte importante da amostra encontra-se com risco aumentado de cair.

A prevalência de dor na população idosa é geralmente alta. No presente estudo, identificouse que 75,8\% dos indivíduos da amostra apresentaram queixa de dor, semelhante à apresentada no estudo de Brattberg, Parker \& Thorslund, que foi de $72,8 \% .^{30}$ A percepção da dor é subjetiva, com limiares muito diferentes para cada indivíduo. A mensuração da intensidade implica confiar na informação prestada pelo entrevistado, podendo gerar viés na interpretação dos resultados. Grande parte dos idosos relatou sentir dor nos membros inferiores. Alguns estudos demonstram relação entre a presença de dor nos membros inferiores e deterioração do equilíbrio, levando ao aumento do risco de cair. ${ }^{31,32}$ 
As quedas foram mais frequentes no sexo feminino, o que está de acordo com os dados da literatura. ${ }^{33-36}$ Essa constatação poderia ser explicada pelo fato de mulheres possuírem menos massa muscular que homens, apresentarem maior perda de massa óssea após a menopausa, com maior risco de fratura (algumas quedas ocorrem após fraturas espontâneas de membros inferiores) e sua maior exposição a tarefas domésticas, com risco de queda. ${ }^{23}$ Os resultados apontam maior vulnerabilidade no gênero feminino em relação à ocorrência de quedas e sugerem que ações para a prevenção desse evento devem ter especial atenção a esta parcela da população. Adicionalmente, estudos que investigam a ocorrência de quedas devem considerar a influência do gênero no seu delineamento.

Não houve associação entre medo de cair e ocorrência de quedas na amostra avaliada. Observouse que a maioria dos idosos, tanto no grupo com história de quedas quanto no grupo que não caiu no ano anterior, relatou medo de cair. Estudos demonstram que o medo de cair pode ocorrer independentemente da ocorrência de quedas. ${ }^{37}$ $\mathrm{O}$ medo de cair pode restringir as atividades dos indivíduos, levando-os a um declínio funcional acompanhado por diminuição da qualidade de vida e aumento do risco futuro de quedas, dados corroborados por estudo de Dias et al. ${ }^{38}$

Também não foi encontrada associação entre nível de atividade física dos indivíduos e ocorrência de quedas. Outros estudos também demonstraram falta de associação entre essas duas variáveis. ${ }^{29,39}$ Entretanto, há estudos que relatam que, se por um lado a realização de atividade física melhora a capacidade funcional e pode reduzir o risco de quedas, por outro, ela também expõe o indivíduo a situações com risco de quedas. ${ }^{40,41}$ Há também a ocorrência de quedas em idosos com baixo nível de atividade física, que se mostram mais vulneráveis, o que leva ao declínio da força muscular e do controle de equilíbrio corporal. ${ }^{42}$ No entanto, o aumento da prevalência de quedas tanto em idosos com baixos quanto altos níveis de atividade física, caracterizada por uma relação em forma de U, não foi comprovada. A literatura ainda é controversa em relação a esse assunto.
Neste estudo, não se verificou associação entre a ocorrência de quedas e as variáveis "restrição de atividades" e "autopercepção de saúde", o que não está de acordo com alguns estudos anteriores. ${ }^{1,43}$ É possível que variações em relação à forma de mensuração dessas variáveis e ao perfil mais ativo da amostra do presente estudo justifiquem tal divergência. ${ }^{44,45}$ Ferreira et al. ${ }^{46}$ e Lang, Michel \& Zekry ${ }^{47}$ propõem que a redução da atividade física com o avançar da idade colabora com o declínio da reserva funcional dos sistemas cardiovasculares, respiratórios e musculoesqueléticos. Esses fatores contribuem para a maior percepção de esforço nas atividades de vida diária. Aumentase, assim, a probabilidade de evitar os esforços, levando a um decréscimo fisiológico progressivo e constante da capacidade de reserva funcional, havendo aumento da sarcopenia e da restrição de atividade física.

Alguns estudos mostram que o idoso com limitações em suas atividades de vida diária secundárias ao medo de cair e que realizam as atividades de forma mais lenta e cuidadosa apresentam pior percepção de saúde. ${ }^{38,48}$ Os idosos com autopercepção da saúde ruim apresentam comprometimento progressivo da capacidade funcional ao longo do tempo, desenvolvendo medo de futura queda, mesmo em pessoas sem história de quedas. ${ }^{36}$

De acordo com os resultados do presente estudo, os pontos de corte dos testes de equilíbrio e mobilidade investigados deveriam ser mais baixos em relação ao proposto pela literatura, para avaliar melhor a população recrutada. ${ }^{49}$ No presente estudo, observou-se que nos testes sentado para de pé e step teste, os pontos de corte que ofereceram maior sensibilidade e especificidade foram 8,5 e 7,5 segundos, respectivamente. No estudo de Tiedemann et al., ${ }^{49}$ os pontos de corte foram 12 e 10 segundos. Em relação ao teste da velocidade de marcha, foi demonstrado que velocidade inferior a $0,7 \mathrm{~m} / \mathrm{s}$ foi o melhor preditor para ocorrência de quedas. Novamente, observa-se que para os idosos do presente estudo, o ponto de corte mais adequado seria inferior a este $(0,98 \mathrm{~m} / \mathrm{s}) .{ }^{22}$ É possível que esse 
resultado esteja relacionado a um melhor nível funcional da amostra, devido à prática regular de atividades físicas. Observa-se que o desempenho dos idosos nos testes foi alto em relação a outros estudos. Como a amostra foi composta por idosos que participavam de grupos de convivência e que apenas $7,8 \%$ não realizavam atividade física regular, é possível que este seja um fator que tenha influenciado os resultados dos testes. Entretanto, há diferenças metodológicas entre os estudos, como o delineamento, que podem ter influenciado nesta divergência. Em relação aos valores de sensibilidade e especificidade alcançados, os valores obtidos por Tiedemann et al. ${ }^{13}$ foram próximos aos deste estudo.

O estudo atual apresenta a limitação de ser baseado numa análise retrospectiva da ocorrência de quedas. Assim, é possível haver um viés de memória em relação ao relato da ocorrência de quedas. Adicionalmente, a capacidade de predição dos testes não pode ser verificada. Entretanto, o estudo trouxe informações importantes a respeito da necessidade da adequação dos pontos de corte para idosos brasileiros ativos e independentes na comunidade. A amostra avaliada está em acompanhamento também de forma longitudinal e os dados prospectivos serão analisados futuramente.

\section{CONCLUSÃO}

De acordo com os resultados do estudo, apenas ser do gênero feminino associou-se com a história pregressa de quedas. Esse dado sugere que as associações entre as variáveis analisadas e a ocorrência de quedas podem variar de acordo com o perfil da amostra estudada. Os resultados também demonstraram ser importante adequar os pontos de corte dos testes de equilíbrio e mobilidade ao perfil da população avaliada. Sem esta adequação, os testes têm sua sensibilidade e especificidade comprometidas na identificação de idosos com risco de cair.

\section{REFERÊNCIAS}

1. Siqueira FV, Facchini LA, Piccini RX, Tomasi E, Thumé E, Silveira DS, et al. Prevalência de quedas em idosos e fatores associados. Rev Saúde Pública 2007;41(5):749-56.

2. Jorge MHPM, Koizumi MS. Gastos governamentais do SUS com internações hospitalares por causas externas: análise no Estado de São Paulo, 2000. Rev Bras Epidemiol 2004;7(2):228-38.

3. Fabrício SCC, Rodrigues RAP, Junior MLC. Causas e conseqüências de quedas em idosos atendidos em hospital público. Rev Saúde Pública. 2004;38(1):93-9.

4. Murphy SL, Williams CS, GILL TM. Characteristics associated with fear of falling and activity restriction in community-living older persons. J Am Geriatr Soc 2002;50(3):516-20.

5. Maia BC, Viana PS, Arantes PMM, Alencar MA. Consequências das quedas em idosos vivendo na comunidade: revisão sistemática. Rev Bras Geriatr Gerontol 2011;14(2):381-93.

6. Tinetti ME, Speechley M, Ginter SF. Risk factors for falls among elderly persons living in the community. New Eng J Med 1988;319(26):1701-7.

7. Scott V. World Heath Organization Report: Prevention of Falls in Older Age [Internet]. Geneva: WHO; 2007 [acesso em 23 Out 2013]. Disponivel em: http://www.who.int/ageing/ projects/5.Intervention, $\% 20$ policies $\% 20$ and $\% 20$ sustainability $\% 20$ of $\% 20$ falls $\% 20$ prevention.pdf.

8. American Geriatrics Society; British Geriatrics Society. Summary of the Updated American Geriatrics Society/British Geriatrics Society clinical practice guideline for prevention of falls in older persons. J Am Geriatr Soc 2011;59(1):148-57.

9. Moreland J, Richardson J, Chan D, O’Neill J, Bellissimo A, Grum R, et al. Evidence-based guidelines for the secondary prevention of falls in older adults. Gerontology 2003;49(2):93-116.

10. Alexander NB. Postural control in older adults. J Am Geriatr Soc 1994; 42(1):93-108.

11. Piirtola M, Era P. Force Platform Measurements as Predictors of Falls among Older People: a review. Gerontology 2006;52:1-16.

12. Muir SW, Berg K, Chesworth B, Klar N, Speechley M. Quantifying the magnitude of risk for balance 
impairment on falls in community-dwelling older adults: a systematic review and meta-analysis. J Clin Epidemiol 2010;63(4):389-406.

13. Tiedemann A. The development of a validated falls risk assessment for use in clinical practice [tese]. Sydney: University of New South Wales, School of Public Health and Community Mecicine; 2006.

14. Verghese J, Holtzer R, Lipton RB, Wang C. Quantitative gait markers and incident fall risk in older adults. J Gerontol A Biol Sci Med Sci 2009;64(8):896-901.

15. Lebrão ML, Laurenti R. Saúde, bem-estar e envelhecimento: o estudo SABE no Município de São Paulo. Rev Bras Epidemiol 2005;8(2):127-41.

16. Benedetti TRB, Mazo GZ, Barros MV. Aplicação do Questionário Internacional de Atividades Físicas para avaliação do nível de atividades físicas de mulheres idosas: validade concorrente e reprodutibilidade teste/ reteste. Rev Bras Ciênc Mov 2004;12(1):25-33.

17. Benedetti TRB, Antunes PC, Rodriguez-Anez CR, Mazo GZ, Petroski EL. Reprodutibilidade e validade do questionário internacional de atividade física (IPAQ) em homens idosos. Rev Bras Med Esporte 2007;13(1):11-6.

18. Mazo GZ, Benedetti TRB. Adaptação do questionário internacional de atividade física para idosos. Rev Bras Cineantropom Desempenho Hum 2010;12(6):480-84

19. Berg K. Measuring balance in the elderly: preliminary development of an instrument. Physiother Can 1989;41(6):304-11.

20. Portney LG, Watkins MP. Foundations of clinical research: application to practice. Upper Saddle River: Prentice Hall; 2000.

21. Studenski S, Perera S, Patel K, Rosano C, Faulkner $\mathrm{K}$, Inzitari M, et al. Gait speed and survival in older adults. JAMA 2011;305(1):50-8.

22. Shimada H, Suzukawa M, Tiedemann A, Kobayashi K, Yoshida H, Suzuki T. Which neuromuscular or cognitive test is the optimal screening tool to predict falls in frail community-dwelling older people? Gerontology 2009;55(5):532-8.

23. Cruz DT, Ribeiro LC, Vieira MT, Teixeira MTB, Bastos RR, Leite ICG. Prevalência de quedas e fatores associados em idosos. Rev Saúde Pública 2012;46(1):138-46.

24. Siqueira FV, Facchini LA, Silveira DS, Piccini RX, Tomasi E, Thumé E, et al. Prevalence of falls in elderly in Brazil: a countrywide analysis. Cad Saúde Pública 2011;27(9):1819-26.
25. Coimbra IB, Pastor EH, Greve JMDA, Puccinelli MLC, Fulller R, Cavalcanti FS, et al. Consenso brasileiro para o tratamento osteoartrite (artrose). Rev Bras Reumatol 2002;42(6):371-4.

26. Flores LM, Mengue SS. Uso de medicamentos por idosos em região do sul do Brasil. Rev Saúde Pública 2005;39(6):924-9.

27. Ribeiro AQ, Rozenfeld S, Kelin CH, César CC, Acurcio FA. Inquérito sobre uso de medicamentos por idosos aposentados, Belo Horizonte, MG. Rev Saúde Pública, J. Public health 2008;42(4):724-32.

28. Lojudice DC, Laprega MR, Rodrigues RAP, Rodrigues AL Júnior. Quedas de idosos institucionalizados: ocorrência e fatores associados. Rev Bras Geriatr Gerontol 2010;13(3):403-12.

29. Benedetti TRB, Binotto MA, Petroski EL, Gonçalves LHT. Atividade física e prevalência de quedas em idosos residentes no sul do Brasil. Rev Bras Geriatr Gerontol 2008;11(2):145-54.

30. Brattberg G, Parker MG, Thorslund M. The prevalence of pain among the oldest old in Sweden. Pain 1996;67(1):29-34.

31. Jadelis K, Miller ME, Ettinger WH junior, Messier SP. Strength, balance, and the modifying effects of obesity and knee pain: results from the Observational Arthritis Study in Seniors (OASIS). J Am Geriatr Soc 2001;49(7):884-91.

32. Hurley MV, Rees J, Newham DJ. Quadriceps function, proprioceptive acuity and functional performance in healthy young, middle-aged and elderly subjects. Age Ageing 1998;27:55-62.

33. Fried LP, Tangen CM, Walston J, Newman AB, Hirsch C, Gottdiener J, et al. Frailty in older adults: evidence for a phenotype. J Gerontol A Biol Sci Med Sci 2001;56(3):146-56.

34. Foldavari, Clark M, Laviolette LC, Bernstein MA, Kaliton D, Castaneda C, et al. Association of muscle power with functional status in community-dwelling elderly women. J Gerontol A Biol Sci Med Sci 2000;55(4):192-9.

35. Campbell AJ, Borrie MJ, Spears GF. Risk factors for falls in a community-based prospective study of people 70 years and older. J Gerontol 1989;44(4):112-17.

36. Perracini MR, Ramos LR. Fatores associados a quedas em uma coorte de idosos residentes na comunidade. Rev Saúde Pública 2002;36(6):709-16

37. Lopes KT, Costa DF, Santos LF, Castro DP, Bastone AC. Prevalência do medo de cair em uma população de idosos da comunidade e sua correlação com mobilidade, equilíbrio dinâmico, risco e histórico de quedas. Rev Bras Fisioter 2009;13(3):233-9. 
38. Dias RC, Freire MTF, Santos EGS, Vieira RA, Dias JMD, Perracini MR. Características associadas à restrição de atividades por medo de cair em idosos comunitários. Rev Bras Fisioter 2011;15(5):406-13.

39. Teixeira LF. Nível de atividade física e quedas em idosos da comunidade: um estudo exploratório [dissertação]. São Paulo: Universidade Cidade de São Paulo; 2011.

40. Yardley L, Beyer N, Hauer K, Kempen G, PiotZiegler C, Todd C. Development and initial validation of the Falls Efficacy Scale-International (FES-I). Age Ageing 2005;34(6):614-9.

41. Cabrera MA, Wajngarten M, Gebara OC, Diament J. [Relationship between body mass index, waist circumference, and waist-to-hip ratio and mortality in elderly women: a 5-year follow-up study]. Cad Saúde Publica 2005; 21:767-75. Português.

42. Peeters GM, Van Schoor NM, Pluijm SM, Deeg DJ, Lips P. Is there a U-shaped association between physical activity and falling in older persons? Osteoporos Int 2010;21:1189-95.

43. Delbaere K, Close JC, Mikolaizak AS, Sachdev PS, Brodaty H, Lord SR. The Falls Efficacy Scale International (FES-I): a comprehensive longitudinal validation study. Age Ageing 2010;39(2):210-6.
44. Wilson MM, Miller DK, Andresen EM, Malmstrom TK, Miller JP, Wolinsky FD. Fear of falling and related activity restriction among middle-aged African Americans. J Gerontol Ser A Biol Sci Med Sci 2005;60(3):355-60.

45. Martin FC, Hart D, Spector T, Doyle DV, Harari $\mathrm{K}$. Fear of falling limiting activity in young-old women is associated with reduced functional mobility rather than psychological factors. Age Ageing 2005;34(3):281-7.

46. Ferreira MT, Matsudo SMM, Ribeiro MCSA, Ramos LRR. Health-related factors correlate with behavior trends in physical activity level in old age: longitudinal results from a population in Sao Paulo, Brazil. BMC Public Health 2010;10: 1-10.

47. Lang PO, Michel JP, Zekry D. Frailty syndrome: a transitional state in a dynamic process. Gerontology 2009;55(5):539-49.

48. Silva A, Faleiros HH, Shimizu WAL, Nogueira LM, Nhãn LL, Da Silva BMF, et al. Prevalência de quedas e de fatores associados em idosos segundo etnia. Ciênc Saúde Coletiva 2012;17(8):2181-90.

49. Tiedemann A, Lord S, Sherrington C. The development and validation of a brief performancebased fall risk assessment tool for use in primary care. J Gerontol Ser A Biol Sci Med Sci 2010;65(8):896-903. 